\title{
Evaluation of a Real-Time Hybrid Three-Dimensional Echo and X-Ray Imaging System for Guidance of Cardiac Catheterisation Procedures
}

\author{
R.J. Housden ${ }^{1}$, A. Arujuna ${ }^{1,2}$, Y. $\mathrm{Ma}^{1}$, N. Nijhof ${ }^{3}$, G. Gijsbers ${ }^{3}$, R. Bullens ${ }^{3}$, \\ M. O’Neill ${ }^{1,2}$, M. Cooklin ${ }^{2}$, C.A. Rinaldi ${ }^{2}$, J. Gill ${ }^{2}$, S. Kapetanakis ${ }^{2}$, \\ J. Hancock ${ }^{2}$, M. Thomas ${ }^{2}$, R. Razavi ${ }^{1,2}$, and K.S. Rhode ${ }^{1}$ \\ 1 Division of Imaging Sciences and Biomedical Engineering, \\ King's College London, UK \\ 2 Department of Cardiology, Guy's and St. Thomas' NHS Foundation Trust, \\ London, UK \\ 3 Philips Healthcare, Interventional X-ray, Best, The Netherlands
}

\begin{abstract}
Minimally invasive cardiac surgery is made possible by image guidance technology. X-ray fluoroscopy provides high contrast images of catheters and devices, whereas 3D ultrasound is better for visualising cardiac anatomy. We present a system in which the two modalities are combined, with a trans-esophageal echo volume registered to and overlaid on an X-ray projection image in real-time. We evaluate the accuracy of the system in terms of both temporal synchronisation errors and overlay registration errors. The temporal synchronisation error was found to be $10 \%$ of the typical cardiac cycle length. In 11 clinical data sets, we found an average alignment error of $2.9 \mathrm{~mm}$. We conclude that the accuracy result is very encouraging and sufficient for guiding many types of cardiac interventions. The combined information is clinically useful for placing the echo image in a familiar coordinate system and for more easily identifying catheters in the echo volume.
\end{abstract}

Keywords: Intervention guidance, image fusion, registration, X-ray fluoroscopy, 4D ultrasound.

\section{Introduction}

Minimally invasive cardiac interventions are becoming increasingly feasible with developments in image guidance [1. X-ray fluoroscopy is commonly used for guidance, as it provides real-time images with excellent visualisation of catheters and other devices. However, fluoroscopy provides only a two dimensional (2D) projection image and has poor soft tissue contrast, and so is not sufficient to guide complex procedures. The complementary technology of trans-esophageal echo (TEE) provides real-time volumetric images with good contrast of cardiac anatomy, but is susceptible to artefacts when imaging mechanical devices such 
as catheters. Also, TEE has a limited field of view and therefore requires a skilled cardiologist to provide anatomical context. The combination of the information in X-ray fluoroscopy and 4D TEE is potentially useful for procedure guidance.

We have developed a system in which the TEE volume is registered to and overlaid on the X-ray, allowing simultaneous real-time visualisation of cardiac anatomy and interventional devices. This concept has been proposed previously and the various implementations differ mainly in the way the echo volume is registered to the X-ray. Existing approaches include tracking the TEE probe with an electromagnetic or mechanical tracking device [2 3] and tracking the TEE probe head, or markers attached to the probe head, in the X-ray images [4/5]. In this paper, we present a complete real-time clinical system for hybrid echo/X-ray navigation that was developed from the proof-of-concept methodology in [4. We evaluate the temporal and spatial accuracy of our overlay alignment and present initial clinical experience of our system used in atrial fibrillation (AF) catheter ablation cases and trans-aortic valve implantation (TAVI) procedures.

\section{Methods}

\subsection{System Overview}

The scanning setup comprises a Philips Allura Xper FD10 C-arm X-ray system and a Philips iE33 3D ultrasound system with an X7-2t 3D TEE probe. Data is streamed from each system to a PC running the visualisation software and displayed in real-time. The software displays a $2 \mathrm{D}$ projection view in $\mathrm{X}$-ray image coordinates, overlaid with a volume rendering of the echo volume.

The two views are registered using the projection image of the TEE probe in the X-ray, following the method described in [4. A 3D model of the TEE probe head, acquired from a nano-CT scan, is registered to the X-ray. The registration is initialised manually to approximately the correct position and orientation before running an automatic 3D-2D registration algorithm. Following the initial registration, the automatic registration is repeatedly rerun to track changes in the probe position due to cardiac and respiratory motion. The automatic GPUaccelerated registration updates in this way at a rate of 1-2 Hz. Figure 1 shows a typical X-ray view, the probe model and a registration of the two.

\subsection{Temporal Synchronisation}

The real-time X-ray and echo images are received from different sources and are processed in different ways before being transmitted to the visualisation software. It is important to determine the relative synchronisations of the two data streams in the overlay view. We expect any delay in the X-ray stream to be constant, but the processing applied to the echo volume in the ultrasound machine may depend on various settings, such as imaging depth, which can be changed several times during a clinical procedure.

The temporal synchronisation was determined by imaging a catheter with simultaneous X-ray and echo. The ultrasound machine was set to image in its live 

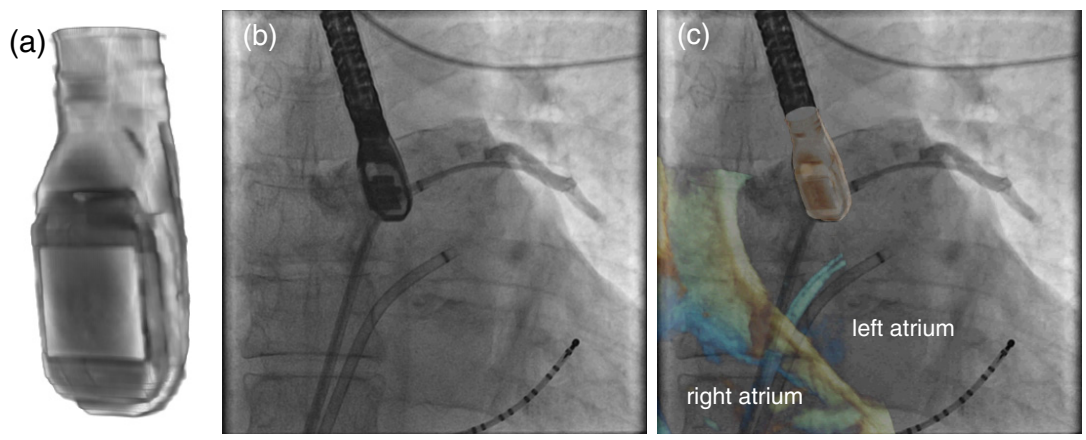

Fig. 1. Automatic overlay registration. (a) TEE probe model from a nano-CT scan of the probe head. (b) X-ray image with the projection of the TEE probe clearly visible. This example is from an AF ablation case. (c) Echo overlay, with the probe model registered to the $\mathrm{X}$-ray by automatic $3 \mathrm{D}-2 \mathrm{D}$ registration.

3D mode. The catheter was given a sharp tap such that the resulting movement was easily detectable in both the X-ray and echo images by sequentially differencing the data. The time difference between the start of movement in the X-ray and echo images provides the relative delay in the overlay view. The delay was measured over the available range of depth settings of the ultrasound scanner (3-26 cm) with 1-3 separate measurements at each depth.

\subsection{Spatial Alignment Accuracy}

As explained above, the overlay registration is achieved by aligning images of the ultrasound probe. The alignment accuracy of the echo volume to the X-ray image is affected by both the accuracy of this probe tracking and the accuracy of the previously calibrated, fixed transformation from the probe to the echo volume. The alignment is particularly susceptible to orientation errors, as even small angular errors can be extrapolated to large misalignments. These alignment errors were measured in phantom and in vivo experiments. The accuracy may also depend on the probe orientation in the projected view. In this paper, the analysis is restricted to probe views typically used in clinical practice.

The alignment error in an overlay view was calculated in terms of the $2 \mathrm{D}$ projection error [6]. This error, $e$, is given by

$$
e=\frac{1}{N} \sum_{i=1}^{N}\left\|P_{i, \text { xray }}-P_{i, \text { echo }}\right\| \frac{D_{\text {source } \rightarrow \text { target }}}{D_{\text {source } \rightarrow \text { detector }}}
$$

where $P_{i \text {,xray }}$ and $P_{i \text {,echo }}$ are the locations of corresponding landmarks defined in the X-ray and echo projections respectively and $N$ is the number of these points 
in the image. The distances $D_{\text {source } \rightarrow \text { target }}$ and $D_{\text {source } \rightarrow \text { detector }}$ account for the magnification in the projected image and their values are known from the C-arm geometry, although the value for $D_{\text {source } \rightarrow \text { target }}$ is known only approximately. The error value is an average of the error at several landmarks in the image so that each overlay view gives one error value. The final error is given in $\mathrm{mm}$.

Phantom Experiment. The phantom comprises a water tank in which two wires are suspended forming a cross. This was imaged by the TEE probe with the ultrasound machine in full volume mode. Corresponding X-ray images were acquired at four different C-arm positions (RAO 54, RAO 24, LAO 22 and PA views) each at high and low dose ( $8 \mathrm{X}$-rays in total). From this data, alignment errors were measured for two registration strategies. The first approach was automatic registration following an approximate manual initialisation. This was done on three different images in each X-ray sequence giving 24 overlay views in which to measure errors. The second approach was a careful manual alignment. Note that this manual alignment considered only the probe, not the echo volume, when optimising the alignment. Again, this was done for 24 overlay views.

Landmarks were defined by manually fitting straight lines to the crossed wires in the X-ray and echo projection image. The crossing points were detected automatically from these lines and landmarks were automatically defined in fixed steps along the lines from the crossing point. This provided up to 13 landmarks per overlay view (minimum 10 where the crossing point was near the edge of the view). $2 \mathrm{D}$ projection errors were calculated between corresponding landmarks and averaged according to (1). Figure 2(a) shows an example of this.
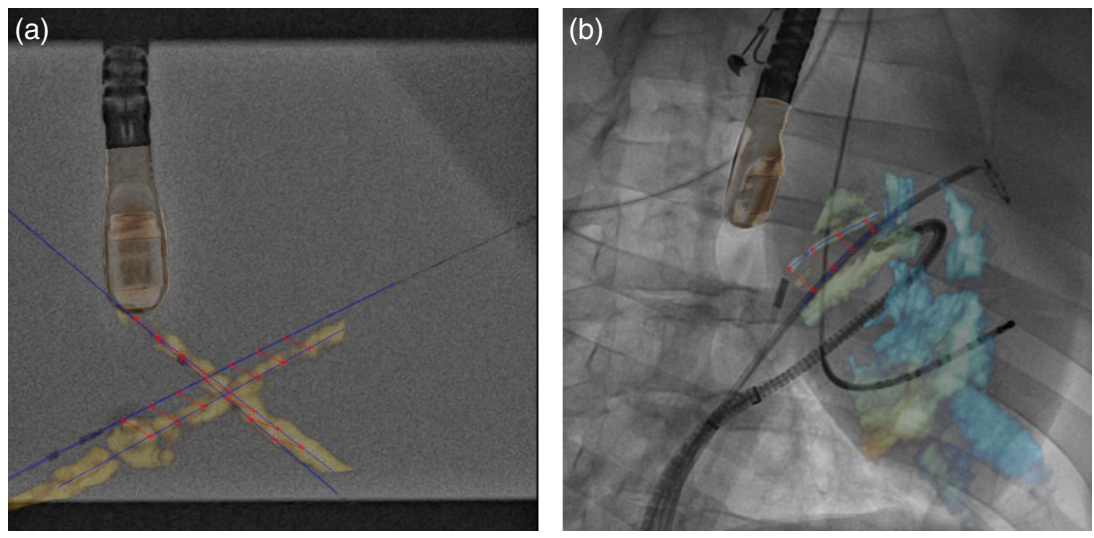

Fig. 2. Error measurement in overlay views. (a) Phantom experiment overlay example. Errors are measured between automatically defined landmarks on straight line models of the crossed wires. (b) Porcine scan overlay example. Errors are measured from landmarks on the echo catheter image to their nearest point on a spline model of the X-ray catheter. 
In Vivo Accuracy. In vivo data to measure accuracy were acquired from a live porcine experiment. Five echo volumes were acquired in full volume mode with the probe in different positions. At each position, three X-ray sequences were recorded in RAO 30, LAO 30 and PA positions with high X-ray dose setting (15 X-ray views in total). Various catheters were inside the heart during the acquisition and these provided convenient targets for measuring alignment errors. Errors were again measured for automatic and manual registrations. These were done at two separate frames in each X-ray sequence (30 measurements), manually selected from the same cardiac and respiratory phases.

Corresponding catheters were manually defined in the echo and X-ray views using Catmull-Rom spline curves. Equally spaced points along the echo curve were automatically selected as echo landmarks. The corresponding X-ray landmark was defined as the closest point on the X-ray curve. The alignment error for each overlay view was again taken as the average of the $2 \mathrm{D}$ errors between landmarks according to (1). An example of these error measurements is given in Fig. 2(b). Average errors were measured using between 3 and 6 landmark pairs per overlay view, depending on the length of catheter visible in the echo image. It should be noted that our approach of measuring to the closest point does not necessarily capture the complete error as there can also be misalignment tangentially to the catheters.

Clinical Cases. The system has been used alongside standard imaging technologies during two types of minimally invasive cardiac procedures: AF ablation and aortic valve replacement (TAVI). During each case, X-ray and echo sequences were recorded independently. Although real-time synchronised visualisation of the live data stream was possible during the procedure, the post-procedure analysis for this paper required that the recorded X-ray and echo data were matched up manually, resulting in only approximately synchronised sequences. 13 such sequences were successfully reconstructed from seven of the nine patients undergoing ablation procedures and six sequences were generated from two TAVI procedures. The errors in each sequence were considered separately.

Unlike the phantom and porcine experiments, the clinical data were analysed in sequences of overlay views over a period of time. There may therefore be additional errors from the real-time probe motion tracking, which may not be able to keep up with sudden or continuous probe movements. Alignment accuracy was measured over the sequences by considering multiple images in each sequence (between 5 and 21 images depending on the sequence length). Statistics for the error over time were calculated for each sequence separately. Errors were calculated using landmarks defined on the catheters and devices in the same way as for the porcine data.

\section{Results and Discussion}

\subsection{Temporal Synchronisation}

Figure 3 shows the results of the time delay experiment. In general, the echo and X-ray are synchronised to within $150 \mathrm{~ms}$. The results show a significant change 
$(p<0.01)$ at around $18 \mathrm{~cm}$ depth. At smaller depth settings, the echo lags the $\mathrm{X}$-ray by $46 \pm 43 \mathrm{~ms}$. At larger depths, the echo leads by $82 \pm 42 \mathrm{~ms}$. This appears to be a sudden change rather than a gradual trend. Therefore, temporal synchronisation could be substantially improved by using a simple pre-calibrated time shift dependent on the depth cut-off value of $18 \mathrm{~cm}$.

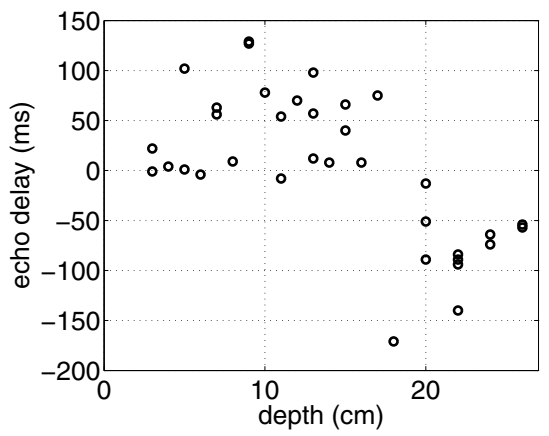

Fig. 3. Relative delay of X-ray and echo. The plot shows the lag of the echo relative to the $\mathrm{X}$-ray over the full range of depth settings.

In terms of clinical implications, the relative delay in the two image streams means that there is a time difference between the display of the catheter locations (most clearly visible in the X-ray image) and the cardiac anatomy (only visible in the echo volume). In a typical cardiac cycle (around $1 \mathrm{~s}$ ), the time difference is on average less than $10 \%$ of the cardiac cycle and at most is within $20 \%$. The visual impact of the delay will depend upon the frame rate of the data streams (maximum 30 frames per second (fps)). For electrophysiology procedures, X-ray frame rate can be as low as 3 fps to minimise radiation dose. The measured delay is then likely to have no impact on ease of navigation using the hybrid approach. The delay is also small compared to the update rate of the $3 \mathrm{D}-2 \mathrm{D}$ registration $(1-2 \mathrm{~Hz})$, although this is only significant when the probe moves substantially.

\subsection{Overlay Alignment Accuracy}

Figure 4 shows the results of the overlay alignment error measurements. Considering first the phantom experiment, the images of the crossed wires are clearly best aligned when positioning the probe manually, which serves as a ground truth. The errors for automatic registration were $6 \mathrm{~mm}$ on average. These results included all registrations even though many would be visually deemed as failures. Detection of registration failure, possibly by use of the similarity metric, would substantially reduce these errors and make them approach the ground truth result. Manual alignment in the porcine data shows similar results to the automatic alignment with average errors of approximately $6 \mathrm{~mm}$. Part of this error will be due to the X-ray and echo data not being recorded simultaneously. 


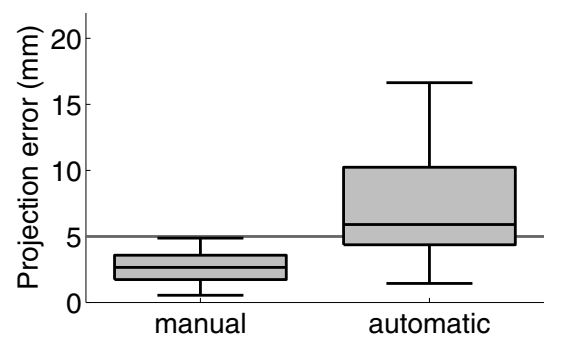

phantom scan

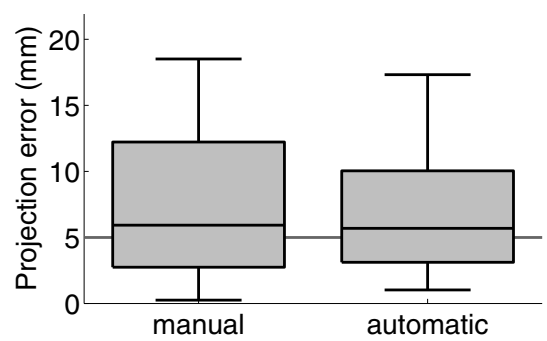

in vivo porcine scan

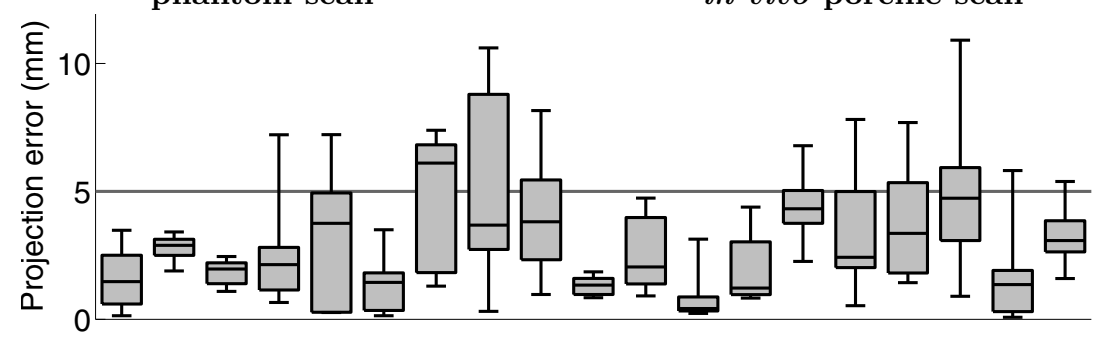

clinical scans

Fig. 4. Overlay alignment errors. The graphs show box plots for the alignment error measurements. Percentiles are at 2.5, 25, 50, 75 and 97.5\%. In the clinical results, the first 13 box plots are for sequences taken from the AF ablation cases and the remaining six are from the TAVI cases. Lines drawn at $5 \mathrm{~mm}$ error are for reference.

The overall average error of the clinical data is $2.9 \mathrm{~mm}$, with a maximum error of $10.9 \mathrm{~mm}$. The accuracy requirement for a clinically useful image guidance system depends on many factors including the patient and the procedure being performed, as discussed in [7]. In general, many of the errors are well below $5 \mathrm{~mm}$ with only a few cases that are worse. Again, detection of registration failures will improve accuracy. The result of $2.9 \mathrm{~mm}$ is very encouraging, especially since it represents a measure of the overall system accuracy in the clinical setting.

A typical overlay from a clinical AF case is shown in Figure 1(c). The clinical results show that the system is sufficiently accurate to guide many different types of cardiac interventions. An advantage of the overlay view is that it helps in interpreting low quality echo images. An echo volume on its own can be difficult to interpret because of its limited field of view and lack of context for the echo coordinate system relative to the patient. Also, catheters and devices tend to produce artefacts in the ultrasound data reducing the clarity of the images. In the overlay view, the echo volume is displayed in a coordinate system that can be more easily related to the patient by the experience of cardiologists working routinely with X-ray fluoroscopy. Also, the highly visible catheters in the X-ray image help with identifying the catheters in the echo and so can be related to the cardiac anatomy via the echo image. In this way, a registered overlay view provides useful information for procedure guidance. 


\section{Conclusions}

In this paper, we have presented a platform for real-time hybrid X-ray fluoroscopy and 3D echo visualisation and have successfully demonstrated it in phantom, animal and clinical experiments. The system exhibits a temporal synchronisation that is less than $10 \%$ of a cardiac period on average. This will not have a significant impact on guidance at the low fluoroscopy frame rates that are often used to minimise radiation exposure. The average $2 \mathrm{D}$ registration error in clinical data is $2.9 \mathrm{~mm}$ which is encouraging for the system's eventual use in clinical guidance. The hybrid approach has the further advantages that it puts the echo into a useful context by positioning it in a familiar coordinate system and the background X-ray helps in identifying catheters and devices in the lower quality echo image. Future work will focus on automatic detection of registration failures in order to maximise clinical robustness.

Acknowledgements. This work was funded by a research grant from Philips Healthcare, Interventional X-ray, Best, The Netherlands.

\section{References}

1. Cleary, K., Peters, T.M.: Image-guided interventions: technology review and clinical applications. Annual Review of Biomedical Engineering 12, 119-142 (2010)

2. Jain, A., Gutierrez, L., Stanton, D.: 3D TEE Registration with X-Ray Fluoroscopy for Interventional Cardiac Applications. In: Ayache, N., Delingette, H., Sermesant, M. (eds.) FIMH 2009. LNCS, vol. 5528, pp. 321-329. Springer, Heidelberg (2009)

3. Ma, Y., Penney, G.P., Bos, D., Frissen, P., Rinaldi, C.A., Razavi, R., Rhode, K.S.: Hybrid echo and X-ray image guidance for cardiac catheterization procedures by using a robotic arm: a feasibility study. Physics in Medicine and Biology 55(13), N371-N382 (2010)

4. Gao, G., Penney, G., Ma, Y., Gogin, N., Cathier, P., Arujuna, A., Morton, G., Caulfield, D., Gill, J., Rinaldi, C.A., Hancock, J., Redwood, S., Thomas, M., Razavi, R., Gijsbers, G., Rhode, K.: Registration of 3D trans-esophageal echocardiography to X-ray fluoroscopy using image-based probe tracking. Medical Image Analysis 16(1), 38-49 (2012)

5. Lang, P., Seslija, P., Habets, D.F., Chu, M.W.A., Holdsworth, D.W., Peters, T.M.: Three-Dimensional Ultrasound Probe Pose Estimation from Single-Perspective XRays for Image-Guided Interventions. In: Liao, H., Edwards, P.J., Pan, X., Fan, Y., Yang, G.Z. (eds.) MIAR 2010. LNCS, vol. 6326, pp. 344-352. Springer, Heidelberg (2010)

6. van de Kraats, E.B., Penney, G.P., Tomazevic, D., van Walsum, T., Niessen, W.J.: Standardized evaluation methodology for 2-D-3-D registration. IEEE Transactions on Medical Imaging 24(9), 1177-1189 (2005)

7. Linte, C.A., Lang, P., Rettmann, M.E., Cho, D.S., Holmes, D.R., Robb, R.A., Peters, T.M.: Accuracy considerations in image-guided cardiac interventions: experience and lessons learned. International Journal of Computer Assisted Radiology and Surgery 7(1), 13-25 (2012) 